\title{
Nota Técnica: \\ El rol de las investigadoras en el sector de la construcción
}

\author{
Technical Note: \\ The role of women in construction research
}

\begin{abstract}
Recepción/Received: 28-XII-09
Aceptación/Accepted: 4-I-10

Publicado online/Online publishing: 9-II-10
\end{abstract}

$\underline{\text { A. Gómez }}^{(*)}$, E. Arrizabalaga(*), B. Sánchez(*)

RESUMEN

La participación equitativa de mujeres y hombres en la investigación científica y el desarrollo tecnológico (I+D) es todavía un desafío por alcanzar en la Unión Europea. Las mujeres europeas siguen estando infra-representadas en este espacio, especialmente en los sectores industriales, a pesar de que en la mayoría de los países miembros la proporción de graduadas excede a la de graduados varones. Esta comunicación presenta cómo es la situación de las investigadoras en el sector de la construcción en Europa, centrando la atención en el papel que juegan en la I+D de este sector, su influencia, perspectivas de futuro, retos y oportunidades. Se presentan los principales resultados soportados por un extensivo trabajo de campo cofinanciado por la Comisión Europea, que muestran una situación de importante desigualdad. Este trabajo ha originado la propuesta de un conjunto de recomendaciones dirigidas a fortalecer la presencia femenina en el ámbito de la I+D en la construcción, e impulsar medios específicos que favorezcan la inhibición de las dificultades que encuentran las mujeres en su profesión científica. Más que nunca, en estos momentos de recesión económica, será necesario invertir en capital humano para la $I+D$, permitiendo que tanto las mujeres como los hombres puedan desplegar plenamente su potencial.

Palabras clave: investigadoras en la construcción, mujer y ciencia, I+D en la construcción, equidad de género en la I+D.

\section{SUMMARY}

Equal participation of women and men in industrial research is still a major challenge for the European Union. In most European countries, female graduates proportionately outnumber male graduates. However, scientific research and technological development remain male-dominated, especially in industry. Construction is one of the most important industrial sectors in terms of economic growth and employmet, but very little is known about the role of women in construction research and the factors that are hindering a more equal presence of women and men. As result of an extensive field work undertaken at European level, this paper offers a picture of the current situation of women in construction research that shows evidences of important inequalities. This work has allowed the development of recommendations for empowering women in construction research, addressing the opportunities for moving towards gender equality in this field. To invest in human capital for research is seen as an outstanding necessity within construction sector, and both women and men should fully develop their potential.

Keywords: Women in construction research, women and science, construction research, gender equality in research.

(*) LABEIN-Tecnalia (Bilbao, España).

Persona de contacto/Corresponding author: araceli@labein.es 


\section{ANTECEDENTES}

El sector industrial juega un papel fundamental en la investigación, el desarrollo tecnológico y la innovación en Europa. El Consejo Europeo estableció en la Cumbre de Barcelona que la meta de asignar el 3\% del PIB a la I+D para 2010 se conseguiría sólo si la industria contribuyera con dos tercios de las nuevas inversiones, lo que significaría doblar la inversión industrial en investigación. Sin embargo, tanto el gasto público como la contribución industrial a la I+D en la Unión Europea sigue estando muy por debajo de los índices que mantienen tanto Japón como los Estados Unidos. Se hace necesario, por lo tanto, continuar promoviendo la investigación en el sector industrial europeo.

En ámbitos como la I+D, las mujeres siguen estando infra-representadas. A pesar de que en la mayoría de los países europeos la proporción de mujeres graduadas excede a la de graduados varones, la investigación científica y el desarrollo tecnológico persisten en su sesgo masculino, especialmente en los sectores industriales. Las mujeres suponen el 30\% del personal investigador en el sector público (universidades y centros públicos de investigación), pero sólo el $15 \%$ del personal investigador en la industria (1). La pérdida desproporcionada de mujeres en las profesiones científicas debe ser considerada con prioridad a fin de evitarla. Su participación en la innovación, la creatividad y la ciencia no está en sintonía con su presencia en los puestos de mayor responsabilidad o de toma de decisiones (2). La participación equitativa de mujeres y hombres en la investigación industrial es todavía un desafío fundamental para la Unión Europea.

\subsection{Excelencia científica en Europa: igualdad en ciencia y tecnología}

La Unión Europea ha experimentado un progreso significativo hacia la igualdad entre hombres y mujeres en cuanto a la legislación, los métodos de transversalidad de género, los programas comunitarios y el diálogo social. Es de reconocer el creciente interés por el tema de mujer y la ciencia, más en particular, por la escasa representación de las mujeres en las carreras científicas y el desproporcionado abandono de sus profesiones científicas que hace que su capacitación y sus conocimientos se vean desperdiciados. Durante la última década se han puesto en marcha diversas iniciativas y grupos de trabajo al amparo de la propia Comisión Europea, entre las que cabe reseñar:

- Comunicación de la CE Mujeres y ciencia: movilizar a las mujeres en beneficio de la investigación europea (1999), con un plan de acción para fomentar la igualdad entre los sexos en la ciencia (3).

- Informe ETAN (2000): encargado a la red European Technology Assessment Network, sobre mujer y ciencia en la UE (4).
- Grupo Helsinki de Mujer y Ciencia (nov. 1999): promueve la discusión e intercambio de experiencias sobre medidas y políticas ideadas y aplicadas con el fin de fomentar la participación de las mujeres en las carreras científicas y la investigación (5).

- Iniciativa WIR, Women in Industrial Research: lanzada por la Dirección General de Investigación de la Comisión Europea en 2001 para mejorar la calidad de las estadísticas sobre el personal científico y de ingeniería - WIR study (2).

- Informe ENWISE (2004) sobre las científicas del centro y este de Europa y Países Bálticos (6).

- Creada la Unidad de Mujer y Ciencia en la DGR, ahora conocida como Science in Society Unit.

- Estadísticas sobre la I+D en los Estados miembros segregadas por sexo: informes She Figures ${ }^{1}$.

- Estudios de evaluación del impacto de los Programas Marco de Investigación de la Unión Europea [FP5 (8) y FP6 (9)].

- Diversos informes publicados por la Comisión Europea, como Gender and Excellence in the Making (10), generado tras el Congreso Minimising gender bias in the definition and measurement of scientific excellen$c e$, que analiza en qué medida los procedimientos, definiciones y criterios existentes en relación a la excelencia científica son neutrales respecto al género.

- EPWS, Plataforma Europea de Mujeres Científicas, creada a final de 2005 para apoyar el trabajo del conjunto de redes nacionales, europeas e internacionales de científicas existentes (11).

Todos estos esfuerzos compartidos, junto a la adopción de la transversalidad de género como una estrategia política generalizada, no sólo han mejorado la situación y los derechos de las mujeres, sino que también han apoyado el desarrollo económico y social de nuestra sociedad. Sin embargo, los datos muestran la persistencia de una baja representación de las mujeres en la I+D, especialmente en el sector industrial. Se hace imprescindible insistir en la obtención de información e indicadores cualitativos y cuantitativos para el diseño de iniciativas y estrategias dirigidas a paliar las desigualdades y proseguir los esfuerzos para suprimir los obstáculos a la participación plena de las mujeres y los hombres en el ámbito de la investigación.

En este contexto se ha desarrollado el proyecto europeo WOMEN-CORE2, Women in Construction Research

1 Editadas las ediciones She Figures 2003 y 2006 (1), (7); publicados los resultados preliminares de She Figures 2009.

2 WOMEN-CORE es un proyecto de investigación cofinanciado por la CE, DG Research, dentro del $6^{\circ}$ Programa Marco de Investigación (No Contrato 017568). Consorcio: LABEIN-Tecnalia (coordinador), CIREM (España), GESIS-CEWS (Alemania), CIFS (Dinamarca), CVUT-Univ. Politécnica de Praga (República Checa), Universidad de Loughborough (Reino Unido). 
[www.women-core.org], orientado a conocer el rol de las mujeres que trabajan en el ámbito de la I+D en el sector de la construcción en Europa y fortalecer su participación y posición. Basado en un extensivo trabajo de campo, los resultados del proyecto muestran una situación de importante desigualdad, algunos de ellos se presentan de forma resumida en el capítulo 3 . El proyecto formula un conjunto de recomendaciones dirigidas a fortalecer la presencia femenina en este ámbito, y propone medios específicos que favorezcan la inhibición de las dificultades que encuentran las mujeres en su profesión científica en el sector de la construcción.

\section{LA I+D EN LA CONSTRUCCIÓN}

La construcción es uno de los sectores industriales más importantes en términos de crecimiento económico y empleo en Europa, que persiste especialmente segregado por motivos de género. Se enfrenta a los retos de mejorar su bajo nivel de I+D (menos del $1 \%$ de su volumen de negocio se invierte en $\mathrm{I}+\mathrm{D}$ y los costes de "no-calidad" se estiman en el 5-10\% de sus ventas totales) (12) y a superar los cambios guiados por las actuales políticas que inciden en el desarrollo sostenible considerando factores sociales, medioambientales y económicos.

Superar los retos señalados exige, entre otras cosas, un incremento del capital humano dedicado a la investigación, donde sería un desperdicio no contar con una mayor implicación de las mujeres cualificadas (13). En la situación económica actual, aún es más importante movilizar a todos los talentos.

La necesidad de atraer, retener y promover a las mujeres parece menos extendida en este sector que en otros sectores industriales. Mientras que los principales agentes en la construcción están desarrollando acciones coordinadas para estimular la I+D y mejorar la innovación, la escasa presencia femenina no está siendo considerada como una preocupación principal. Se conoce muy poco sobre el verdadero papel de las investigadoras en este sector y sobre los factores que dificultan una presencia de mayor igualdad entre mujeres y hombres. WOMEN-CORE ha tratado de progresar en estas cuestiones con el objetivo de fomentar la participación de las científicas y tecnólogas en la I+D del sector de la construcción en Europa.

\subsection{Mapa de la I+D en el sector de la construcción}

WOMEN-CORE distingue claramente la construcción como industria y la I+D en el sector de la construcción. Adoptando como ámbitos de investigación en este sector, los establecidos por la Plataforma Tecnológica de Construcción Europea, ECTP3 (14), replicados en la Plataforma Tecnológica Española de Construcción (15), se consideran siete áreas estratégicas de intervención: cuatro áreas verticales enfocadas a los segmentos específicos de la industria de construcción y tres a áreas horizontales que ayudan a integrar y armonizar los diferentes enfoques, representadas en la Figura 1.

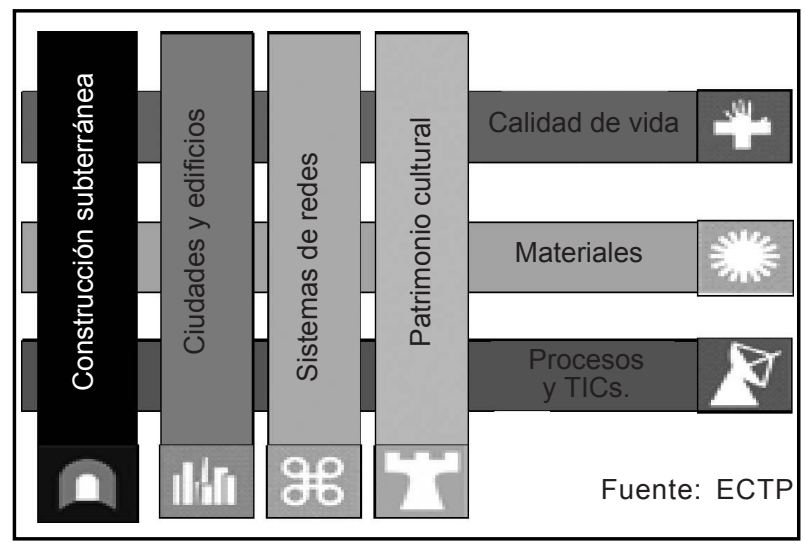

Figura 1. Áreas estratégicas de I+D en el sector de la construcción.

La Tabla 1 muestra las disciplinas académicas relacionadas con las áreas anteriores.

Tabla 1

Disciplinas académicas relacionadas con la I+D en construcción.

- Arquitectura

- Planificación urbana

- Mecánica incluyendo mecánica fluida y dinámica

- Estructuras de edificación (materiales estructurales, estructura de acero, estructuras de madera, estructuras de albañilería)

- Infraestructura y ordenación de las aguas

- Infraestructura y organización del transporte

- Ingeniería ambiental

- Construcción y gestión económica

- Física de la edificación (acústica, prestaciones térmicas y ahorro energético, iluminación)

- Tecnología y organización de la construcción

- Tecnologías de la Información y de las Comunicaciones (TICs)

- Calefacción, refrigeración, ventilación, electricidad y redes

- Geotecnia y estructuras subterráneas

Debido al carácter interdisciplinar de la I+D en construcción, existen muchas otras disciplinas periféricas relacionadas con estas áreas de investigación que han sido también consideradas.

3 ECTP, European Construction Technology Platform, lanzada en los albores del FP7 al amparo de la Comisión Europea con el objeto de promover la I+D del sector [http://www.ectp.org] 
Se han identificado alrededor de 1.500 instituciones en Europa que se dedican a la investigación en el sector de la construcción, clasificándolas en cuatro categorías principales: instituciones de Educación Superior, centros de investigación, empresas (PYMEs y grandes empresas) y organismos públicos.

\subsection{Marco metodológico}

El modelo metodológico utilizado en la investigación ha combinado diversos métodos y técnicas de recopilación y análisis de datos, y métodos de síntesis y evaluación, basados en tres ejes principales: a) análisis estadísticos de fuentes de información existentes; b) trabajo de campo para explorar nuevas fuentes de información; c) exploración de los contenidos de investigación.

Entre las múltiples fuentes consultadas cabe señalar las relacionadas con el personal cualificado para ejercer en la I+D en construcción (EUROSTAT-Educación, Encuesta de I+D, Encuesta Laboral y de la Estructura Salarial), bases de datos de proyectos de I+D financiados por la UE, de patentes (EPO, Oficina Europea de Patentes) y de publicaciones científicas. El trabajo de campo, apoyado en encuestas a individuos e instituciones, entrevistas personales y casos de estudio, ha tenido un especial protagonismo: encuesta a personal investigador y no investigador en el sector (4.000 respuestas analizadas); encuesta a instituciones de I+D (225 instituciones); más de 120 entrevistas a personal investigador y experto en construcción, a personas con autoría en patentes de los sectores de construcción, química y farmacia y entrevistas a editores/as de revistas científicas. Adicionalmente, se han evaluado 84 programas europeos de mentorización y unas 70 redes profesionales relacionadas con el sector.

El equipo de proyecto ha contado con el apoyo de un Comité Asesor externo (Advisory Group) conformado por personas expertas en el sector y en temas de igualdad. Tanto los métodos utilizados como los resultados que se han ido obteniendo han sido sometidos a debate por el Comité Asesor y en seminarios nacionales organizados en los cinco países de las instituciones participantes en el proyecto WOMEN-CORE (Alemania, Dinamarca, España, Reino Unido y República Checa).

\section{RESULTADOS}

\subsection{Presencia femenina en la I+D del sector de la construcción}

Con respecto a la Educación Superior, en 2004 las mujeres representaban un $40,7 \%$ de las personas licenciadas y máster, y un 33,9\% de las doctoras, en disciplinas relacionadas con el sector de la construcción, tal como indica la Figura 2.

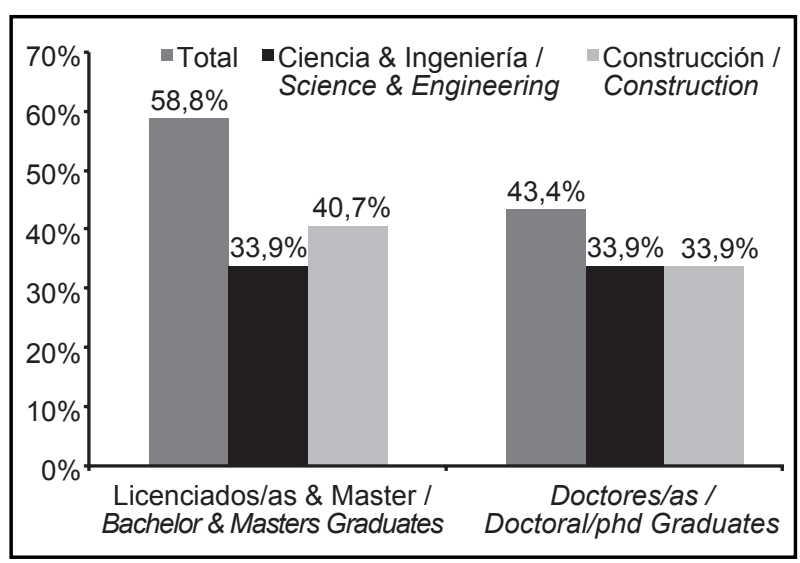

Figura 2. Proporción de mujeres graduadas en la UE27, 2004.

Estos datos muestran la persistencia de una segregación horizontal y vertical en la Educación Superior, pero también que la presencia de las mujeres en las disciplinas relacionadas con la construcción es mayor que la que se encuentra en la ciencia y la ingeniería en su conjunto. Asimismo, respecto a 6 años atrás, la evolución ha sido positiva, con un incremento en la proporción femenina de 3,4 puntos porcentuales (p.p.) en licenciadas y máster, y 6 p.p. en doctoras.

La segregación vertical y horizontal son también rasgos en las profesiones investigadoras del sector de la construcción, tal como indican los siguientes resultados encontrados:

- En 2005, la proporción de investigadoras en el sector de la construcción era sólo del 19\%, con un ligero ascenso de 2,9 p.p. respecto al año 2000.

- Según datos de 2006, la Plataforma Tecnológica de Construcción Europea (ECTP) sólo contaba con un 18\% de mujeres entre el total de sus miembros, estando mejor representadas en las Administraciones públicas (28\%) y en los centros de investigación (23\%), y con una presencia especialmente baja en las empresas (15\%). Teniendo en cuenta las distintas áreas estratégicas de I+D, la participación de las mujeres en la ECTP era variada: las áreas de Patrimonio cultural, Redes y Materiales ofrecían una mejor presencia femenina con un $13 \%, 11 \%$ y $10 \%$, respectivamente. Por el contrario las mujeres estaban peor representadas en las áreas más puramente de ingeniería como Construcciones subterráneas (5\%) y Ciudades y edificios (6\%). Se ha experimentado una cierta mejoría en cuanto a la participación de las mujeres en la ECTP en los dos últimos años.

- Existe también una fuerte segregación vertical en las profesiones investigadoras en construcción, con una muy pequeña proporción de mujeres en puestos de responsabilidad y de liderazgo.

- Es destacable la segregación horizontal respecto a las áreas de I+D: las mujeres trabajan en mayor proporción en instituciones que desarrollan actividades en arquitectura y planificación urbana, mientras que los 
varones lo hacen en mayor proporción en instituciones dedicadas a investigar sobre construcción subterránea y los procesos y las TICs.

La participación femenina en proyectos de I+D financiados por la Comisión Europea4, también es escasa, tal y como muestra la Figura 3: de media, sólo el $8,7 \%$ de las personas que participan en ellos son mujeres, el 10,1\% coordinando los proyectos y el $8,6 \%$ como directoras científicas.

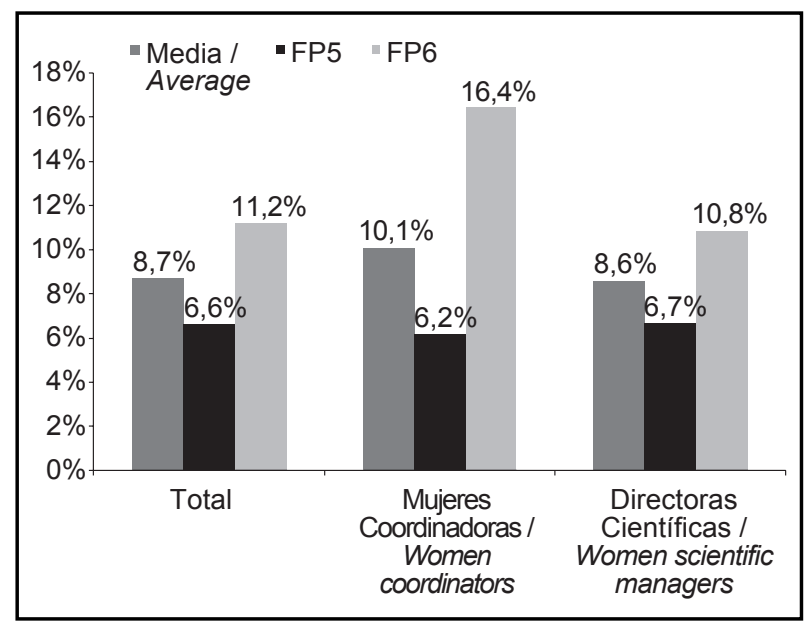

Figura 3. Proporción de mujeres participantes en proyectos europeos de I+D en construcción, 1998-2006.

Se observa un incremento de la participación femenina en los proyectos europeos entre el $5^{\circ}$ y $6^{\circ}$ Programa Marco de $I+D$, con mayor incremento en la coordinación de proyectos, y una gran variación entre los países participantes en el proyecto, donde nuestro país presenta el mejor incremento, mientras que el Reino Unido experimenta un descenso, tal como muestra la Figura 4.

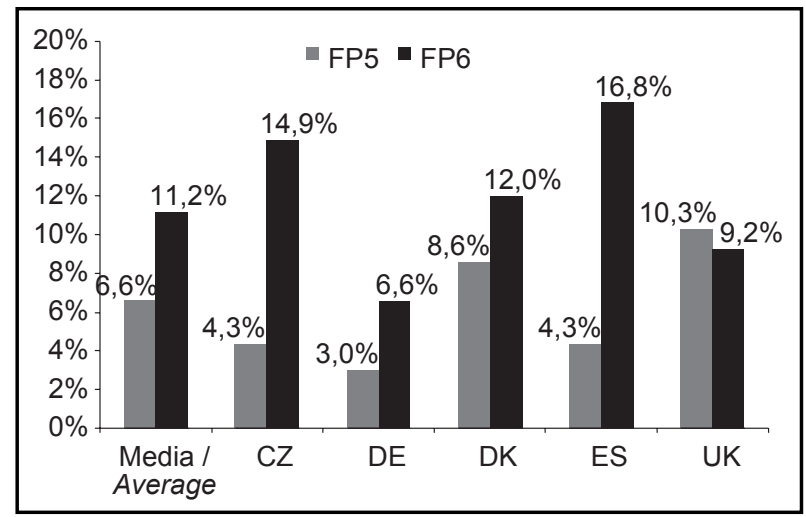

Figura 4. Proporción de mujeres participantes en proyectos europeos de I+D en construcción por países.

4 Identificados y analizados 497 proyectos financiados por la UE, 308 del $5^{\circ}$ Programa Marco (1998-2002) y 189 del $6^{\circ}$ Programa Marco (2003-2006).
La autoría de las mujeres en patentes ${ }^{5}$ relacionadas con el sector de la construcción es especialmente escasa. En 2004, sólo el 4,9\% de las patentes de este sector correspondían a mujeres. Si bien es cierto que patentar es menos común en el sector de la construcción comparado con los sectores químico o farmacéutico, la participación femenina en patentes era muy baja en todos ellos a la vista de lo indicado en la Figura 5 (sector químico $11,7 \%$, sector farmacéutico $21,4 \%)$.

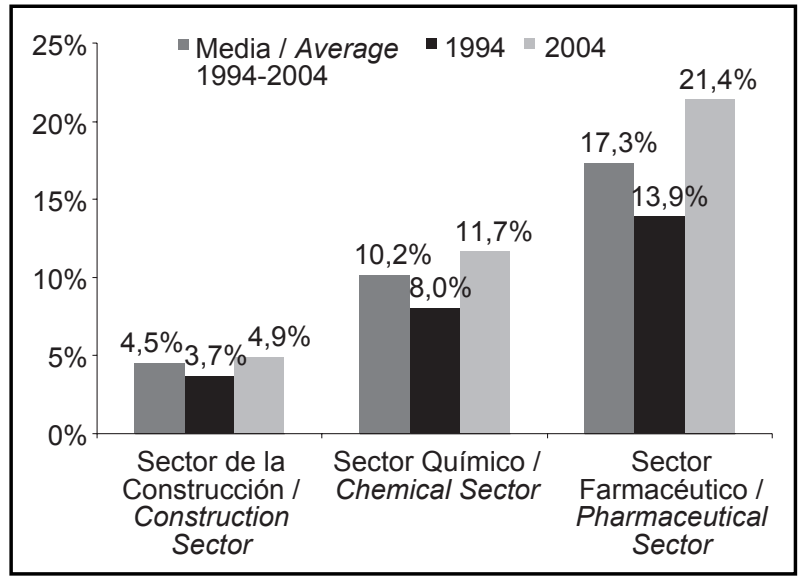

Figura 5. Proporción de mujeres con patentes en EU25, 1994-2004.

Por otra parte, se ha observado un ligero incremento de 1,2 p.p. en la proporción media de patentes de mujeres entre 1994 y 2004, con variaciones entre países. Nuestro país experimenta una situación algo mejor, donde el porcentaje de mujeres patentadoras evolucionó del 6,4\% en 1994 al 8,1\% en 2004. Sin embargo, Alemania, con el mayor número de patentes en Europa entre 1994-2004, registra la peor situación, donde sólo un 4,4\% de ellas son de autoría femenina.

El estudio realizado permite concluir que patentar no parece una prioridad para progresar profesionalmente en el ámbito de la I+D en Europa tanto para hombres como para mujeres. La impresión común es que las patentes representan mayor valor para las instituciones que para el propio personal investigador.

Finalmente, se indican algunos resultados obtenidos sobre la participación femenina en publicaciones científicas del sector de la construcción 6 , muy valoradas en el

5 Clasificada la BBDD European Patent Office (EPO) por sexo, país, área de tecnología e industria. Fue necesario crear la BBDD First Name Database, FNDB (46.619 nombres propios) para esa clasificación.

6 Analizados 918 artículos de 20 revistas científicas en construcción de 2000 y 2003. 
entorno de la $\mathrm{I}+\mathrm{D}$ y percibidas como un factor relevante para progresar profesionalmente, especialmente en el mundo académico, cediendo en prioridad para el personal investigador de la industria.

En 2003 , el $21,7 \%$ de los/as autores/as que publicaron en revistas científicas de construcción eran mujeres. La mayoría de los/as autores/as provenían de la Universidad (78\% en 2003). Una vez más, se observan variaciones significativas entre países, pero en todos ellos aumentaron el número de autoras entre 2000 y 2003, tal como muestra la Figura 6 (incremento de 7 p.p. de media).

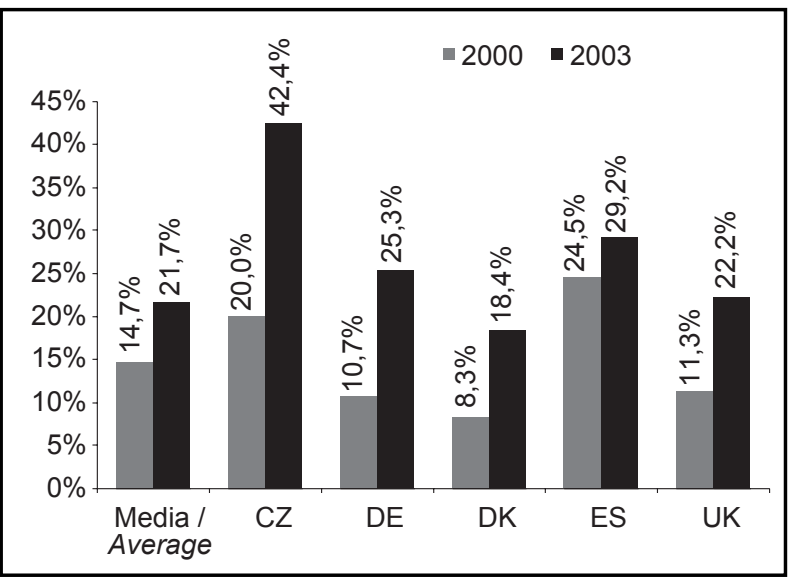

Figura 6. Proporción de autoras en publicaciones de revistas científicas 2000-2003, por países.

Asimismo, se aprecian grandes variaciones en la autoría de las publicaciones dependiendo de las revistas científicas. Se han visto evidencias de que las mujeres pueden tener más éxito en publicar en temas catalogados como soft dentro del sector de la construcción (interiorismo, diseño arquitectónico, medio ambiente), que en aquellos de más pura ingeniería.

\subsection{Políticas de igualdad en las instituciones de investigación}

Las instituciones de Educación Superior (IESs) y los centros de investigación (CCII) presentan mayor presencia femenina en la I+D que las empresas y muestran mejor perfil de medidas de igualdad, especialmente las IESs (prácticas encaminadas a facilitar la conciliación entre vida laboral y privada, políticas de retención y de promoción, etc.). La atención prestada por las instituciones a los aspectos de igualdad refleja en cierta manera la proporción del personal investigador en cada categoría. En general, son los centros de investigación los que disponen de mejores políticas de retención de mujeres investigadoras en construcción con una mayor proporción de investigadoras senior y en puestos de responsabilidad.
Respecto a los patrones de liderazgo, el estudio refleja que la proporción de mujeres decrece según se asciende en los puestos decisorios en todos los tipos de instituciones, siendo las empresas de construcción las que presentan una mayor disminución. Se observan indicios de una relación directa entre la proporción de mujeres en puestos de responsabilidad y la proporción de mujeres en puestos junior en esas instituciones. En relación a las condiciones laborales, es el sector académico el que presenta los salarios más bajos y una mayor temporalidad en los contratos. Existen importantes diferencias salariales entre mujeres y hombres en los distintos tipos de instituciones, diferencias algo mayores en las empresas.

Las IESs presentan un mejor perfil en lo que respecta a medidas de conciliación, tanto en el grado de implantación, como en el mayor número de medidas para favorecer la igualdad. Estas medidas están siendo implantadas en todas las categorías de instituciones, pero con una extensión desigual que varía en función del tipo de medidas y el tipo de institución. El horario de trabajo flexible, el teletrabajo y la mentorización informal son las medidas de conciliación prevalentes. Las cuotas apenas son consideradas.

La mentorización se aprecia como un instrumento muy eficaz para promover el desarrollo profesional en la investigación en construcción, con beneficio mutuo tanto para las personas mentoras como para las mentorizadas. La mentorización informal es dominante frente a la formal. En Alemania y Reino Unido existen programas de mentorización establecidos (especialmente en el entorno académico, aunque también en algunas grandes empresas), mientras que en España no se conoce la existencia de ninguno.

La influencia de las redes profesionales se percibe de manera muy positiva para la progresión en la carrera investigadora en construcción. Favorecen las oportunidades de colaboración y de trabajo, de mejora salarial, promoción y satisfacción, ganando visibilidad, teniendo la posibilidad de recibir consejos y apoyo profesional, entre otros. Sin embargo, mientras que las organizaciones manifiestan que apoyan y facilitan la participación en redes, el personal investigador señala que no dispone de tiempo suficiente en el entorno de trabajo para participar de sus actividades.

\section{CONCLUSIONES}

El análisis realizado en el proyecto WOMEN-CORE se ha dirigido a tres grandes dimensiones de la I+D del sector de la construcción: el personal investigador, las instituciones que realizan la I+D y el contenido de investigación. Brevemente se presentan las conclusiones más relevantes: 
a) Personal investigador. Se han identificado las principales características de la carrera científica en construcción y las tendencias más relevantes que determinan el desarrollo profesional de las personas investigadoras en construcción. Se han observado diferencias significativas entre hombres y mujeres en cuestiones que pueden tener implicaciones relevantes respecto a la continuidad y el éxito profesional. Se han constatado diferencias de género en aspectos tales como el grado de estabilidad, sueldo, oportunidades de ascenso y experiencia laboral, estando algunos de estos aspectos relacionados probablemente con las interrupciones en la vida laboral debidas a las responsabilidades familiares. Unidas a las desigualdades a nivel de las organizaciones, hay otros factores que no ayudan a eliminar las diferencias de género entre el personal investigador: algunos de ellos relacionados con las mujeres mismas, y otros debidos a la falta de apoyo fuera de su trabajo profesional, lo que dificulta el normal desarrollo de su profesión. En concreto, las mujeres parecen carecer de confianza en sí mismas a la hora de afrontar un ascenso u ocupar cargos de mayor responsabilidad. La tradicional división de trabajo también persiste entre el personal investigador y afecta de forma diferente a las oportunidades de promoción de hombres y mujeres.

b) Instituciones que realizan $I+D$. Las instituciones, a través de sus prácticas y estructuras, juegan un papel relevante a la hora de favorecer o no la igualdad entre hombres y mujeres. Han sido identificadas y analizadas las políticas particulares de las organizaciones para atraer, retener y promocionar a las investigadoras, así como las medidas de conciliación laboral y personal implantadas. Para las investigadoras que desarrollan su profesión en las empresas, se observa que es más difícil mantener una progresión lineal en su profesión debido a la inflexibilidad proveniente de los contratos de jornada reducida, el menor nivel de antigüedad y falta de modelos de conducta. En general, las investigadoras se sienten más atraídas por el entorno académico, donde son más comunes otras medidas de promoción, como, por ejemplo, la participación en redes y en programas de mentorización. c) Contenido de investigación. Se están produciendo ciertos cambios en el contenido de la investigación en construcción, apareciendo áreas más multidisciplinares y contenidos científico-tecnológicos con un enfoque más integrador, provocados por las demandas sociales, el respeto al medio ambiente, la eficiencia energética y la sostenibilidad. Estas áreas emergentes pueden constituir una buena oportunidad para mejorar ciertas estructuras en el sector y fomentar la participación femenina en la $\mathrm{I}+\mathrm{D}$.

Las conclusiones del proyecto WOMEN-CORE confirman la necesidad de establecer una serie de recomendaciones para superar las desigualdades encontradas, así como proponer medios específicos que ayuden a las mujeres a superar las dificultades que encuentran en su carrera científica en el sector de la construcción, contribuyendo así al logro de un ambiente de trabajo y una sociedad más igualitaria. Dichas recomendaciones podrían ser integradas en nuevas políticas para la mejora de la igualdad de oportunidades en el sector de la construcción. Los beneficiarios de estas recomendaciones son diferentes grupos: el colectivo con responsabilidad política a nivel europeo, nacional o local; los agentes del sector de la construcción; las instituciones de investigación (instituciones de Educación Superior, centros de investigación públicos y privados, empresas); y el propio personal investigador. Facilitarían la toma de las decisiones en sus estrategias de integración de la igualdad de oportunidades, y en la gestión y evolución de las carreras profesionales en la I+D.

\section{AGRADECIMIENTOS}

Las autoras desean agradecer la financiación recibida de la Comisión Europea bajo el $6^{\circ}$ Programa Marco de I+D para la realización del proyecto WOMEN-CORE (FP6017568). También desean expresar su agradecimiento al Advisory Group y a cada socio del proyecto por su aportación a esta investigación. Las autoras agradecen, además, la financiación recibida de EMAKUNDE-Instituto Vasco de la Mujer, Gobierno Vasco, para el caso de la Comunidad Autónoma del País Vasco, y del Instituto de la Mujer, Ministerio de Igualdad del Gobierno español.

\section{BIBLIOGRAFÍA}

(1) She Figures. European Commission, EUR 20733. Women and Science. Statistics and Indicators, Luxembourg, Office for Official Publications of the European Communities. ISBN 92-894-8229-X.

(2) Meulders, Danièle; Plasman, Robert; Lemière, Severine; Danis, Stephan, O’Dorchai, Síle; Tojerow, Ilan; Jepsen, Maria; Gangji, Amynah; Moreno, David; Caprile, María; Kruger, Karsten: Women in industrial research (WIR) - Analysis of statistical data and good practices of companies. Directorate-General for Research, Science and Science, Luxembourg, Office for Official Publications of the European Communities (2003).

(3) COM (99) 76 final. Comunicación de la Comisión Europea, "Women and Science: mobilising women to enrich European research" (1999). 
(4) Osborn, M.; Rees, T.; Bosch, M.; Ebeling, H.; Hermann, C.; Hilden, J.; McLaren, A.; Palomba, R.; Peltonen, L.; Vela, C.; Weis, D. and Wolh, A.: Science Policies in the EU: promoting excellence through mainstreaming gender equality, A report from the ETAN Expert Working Group on Women and Science, Luxembourg, Office for Official Publications of the European Communities (2000).

(5) Rees, T.: The Helsinki Group National Policies on Women and Science in Europe. DG-Research. Information and Communication Unit. European Commission, Brussels (2002).

(6) EC, EUR 20955. Waste of talents: turning private struggles into a public issue. Women and Science in the ENWISE Countries. A report to the European Commission from the Enwise Expert Group on women scientists in the Central and Eastern European countries and in the Baltic States, Luxembourg, Office for Official Publications of the European Commission (2003).

(7) She Figures. European Commission, EUR 22049. Women and Science Statistics and Indicators. Luxembourg, Office for Official Publications of the European Communities (2006). ISBN 92-79-01566-4.

(8) Laurila, P. and Kerry, Y. (comp.): Synthesis Report-Gender in Research-Gender Impact Assessment of the specific programmes of the Fifth Framework Programme- An overview. European Commission, Directorate General for Research, Luxembourg, Office for Official Publications of the European Commission (2001).

(9) Caprile, M. (ed.); Sánchez, B.; Vallès, N.; Gómez, A.; Potrony, J.; Sixto, E.: Herrera, D.; Oleaga, M.; Amate, M. and Isasa, I.: (2008) Monitoring progress towards Gender Equality in the Sixth Framework Programme. Synthesis Report: Nanotechnologies and nanosciences, knowledge-based multifunctional materials, and new production processes and devices (NMP); Aeronautics and space; Sustainable energy systems; Sustainable surface transport; Euratom. European Commission. Luxembourg, Office for Official Publications of the European Communities (February 2008). ISBN 978-92-79-08506-2.

(10) European Commission, Science and Society: Gender and Excellence in the Making, Luxembourg, Office for Official Publications of the European Commission (2004).

(11) Página web de la Plataforma Europea de Mujeres Científicas, EPWS, European Platform of Women Scientists [http://www.epws.org/]

(12) COM (97) 539 final: Comunicación de la Comisión Europea, "Competitiveness of the construction industry" (4 November 1997).

(13) EC, EUR 21224 (2004): Increasing human resources for science and technology in Europe. European Commission, Luxembourg, Office for Official Publications of the European Communities (2004). ISBN 92-894-8459-4.

(14) Plataforma Tecnológica Europea de Construcción, European Construction Technology Platform, ECTP, Strategic Research Agenda for the European Construction Sector (2005) [http://www.ectp.org/]

(15) Plataforma Tecnológica Española de Construcción, PTEC: Vision 2030, March 2005 [http://www.construccion2030.org/] 